\title{
Suplementação de lipídeos de diferentes fontes em dietas para vacas Jersey na fase inicial de lactação ${ }^{1}$
}

\section{Patrícia Barcellos Costa ${ }^{2}$, Waldyr Stumpf Júnior ${ }^{3}$, José Laerte Nörnberg ${ }^{4}$, Vivian Fischer ${ }^{5}$, Augusto César de Queiroz ${ }^{6}$, Renius Mello}

\author{
${ }^{1}$ Parte do trabalho de Mestrado da primeira autora. Financiado pela EMBRAPA Clima Temperado, PPGZ - UFPel. \\ 2 Médica Veterinária. Doutora em Nutrição e Produção de Ruminantes pela Universidade Federal de Viçosa. \\ ${ }^{3}$ EMBRAPA Clima Temperado-CPACT-Pelotas-RS. \\ ${ }^{4}$ DTCA - UFSM, Santa Maria-RS. \\ ${ }^{5}$ DZO - UFRGS, Porto Alegre-RS. \\ ${ }^{6}$ DZO - UFV, Viçosa-MG. \\ 7 Universidade Federal de Roraima, Doutorando em Zootecnia pela UFV, Viçosa-MG.
}

RESUMO - Objetivou-se avaliar os efeitos da suplementação de lipídeos como sais de cálcio de óleo de palma (GP), farelo de arroz integral + óleo de arroz (FAIO) ou farelo de arroz integral + sebo bovino (FAIS) sobre o consumo de MS, a eficiência produtiva e o balanço energético de vacas leiteiras no terço inicial da lactação. A dieta controle continha 2,9\% EE, enquanto as dietas GP, FAIO e FAIS continham, respectivamente, 5,13; 5,29 e 5,16\% EE. Foram utilizadas oito vacas Jersey, puras de origem, de $2^{a}$ e $3^{\underline{a}}$ ordens de lactação, aos 70 dias de lactação, com média de $420 \mathrm{~kg}$ PV e escore corporal de 3,5 a 4 . O delineamento experimental utilizado foi o quadrado latino $4 \times 4$ duplo, com oito animais, quatro dietas e quatro períodos experimentais de 21 dias (14 dias para adaptação e sete para coleta de amostras para as análises químicas do leite e dos alimentos). A produção de leite corrigida para 3,5\% de gordura (PLC3,5\%) foi maior com a suplementação de lipídeos à dieta. Não houve efeito da suplementação sobre o consumo de MS. A suplementação de lipídeos resultou em maior eficiência produtiva, expressa como PLC3,5\% em relação ao peso metabólico. Independentemente da fonte de lipídeo, não houve efeito das dietas sobre a PLC3,5\%, o consumo de MS e a eficiência produtiva. O uso de diferentes fontes de lipídeos em dietas para vacas leiteiras de alta produção pode ser viável, pois sua inclusão na dieta promoveu aumento na produção leiteira sem alterar o consumo de alimentos.

Palavras-chave: balanço energético, consumo de matéria seca, eficiência de produção, farelo de arroz integral, produção de leite, sais de cálcio de óleo de palma

\section{Fat supplementation from different sources in the diet of Jersey cows in the initial lactation stage}

ABSTRACT - The objective of this research was to evaluate the effects of fat supplementations as calcium salts of palm oil (PF), whole rice bran + rice oil (WRBO) and whole rice bran + bovine tallow (WRBT) on dry matter intake, productive efficiency and energy balance of dairy cows in the beginning of the first third period of lactation. Control diet contained $2.9 \%$ EE, while the diets GP, FAIO and FAIS contained 5.13; 5.29 and 5.16\% EE, respectively. Pure bred Jersey cows of $2^{\text {nd }}$ and $3^{\text {rd }}$ lactation were used, with 70 days in milk, average weight of $420 \mathrm{~kg}$ and body condition of 3.5 to 4 . A replicated 4 x 4 Latin square experimental design was used, with eight cows, four diets and four experimental periods. Each experimental period consisted of 21 days (14 days for adaptation and 7 for sample collection for the chemical analysis of milk and feeds. Milk production of 3.5\% fat corrected milk (FCM 3.5\%) was higher with the fat supplementation to the diet. There was no difference in dry matter intake. Fat supplementation showed better results for productive efficiency expressed as FCM3.5\% in function of metabolic weight. Independently of lipid source, there was not effect of the diets on PLC3.5\%, dry matter intake and productive efficiency, expressed as PLC3.5\% in function of the metabolic weight. The addition of different fat sources to high production dairy cows diets could be viable, because its addition to the diet improved milk yield without alter feed intake.

Key Words: calcium salt of palm oil, energy balance, dry matter intake, milk production, productive efficiency, whole rice bran

\section{Introdução}

A seleção de vacas leiteiras com o objetivo de aumentar a produção de leite tem provocado desbalanço entre consumo e suprimento das necessidades energéticas dos animais. Esse desbalanço ocorre porque a produção de leite na fase inicial do período de lactação é função prioritária desses animais, mesmo quando há consumo insuficiente de energia. 
Nessas circunstâncias, as vacas utilizam suas reservas corporais para satisfazer as necessidades de energia para produção, o que resulta em balanço energético negativo. O consumo máximo de MS em raças européias ocorre entre a 2a e a 6a semana após o pico da lactação, período correspondente à fase da 4 a à 8 a semana pós-parto. Desse modo, as exigências nutricionais para mantença e lactação não são supridas pelo consumo de MS, ocasionando, principalmente, déficit de proteína e energia (Dijk et al., 1983).

A quantidade de energia mobilizada das reservas corporais e utilizada para produção de leite depende do escore corporal, do potencial genético do animal, do número de dias pós-parto e do consumo de alimentos no início da lactação (Hemken, 1970). Aumentar a densidade de energia das dietas pode auxiliar na redução do balanço energético negativo, pois as dietas passam a atender à demanda de energia para produção de leite (Jerred et al., 1990). Palmquist (1988) observou que, em dietas com 8\% de EE na MS, parte da EM (15 a 20\%) foi oriunda de ácidos graxos de cadeia longa (AGCL). O aumento do consumo de AGCL na dieta pode elevar a eficiência metabólica para produção (Kronfeld et al., 1980), pois os ácidos graxos (AG) pré-ingeridos podem ser incorporados diretamente na gordura do leite, diminuindo o gasto de energia para a síntese de AG e poupando energia para outras funções produtivas na glândula mamária (Schauff et al., 1992). Estima-se que a transferência eficiente dos AGCL para o leite varie entre 30 e 50\% em dietas suplementadas com lipídeos (Palmquist, 1984).

Ressalta-se que no estudo dos alimentos e das dietas para vacas leiteiras, para o cálculo correto da eficiência produtiva em relação ao consumo de energia líquida, é importante considerar a estimativa do valor energético dos alimentos. Uma estimativa precisa proporciona cálculos mais corretos das eficiências energética e de produção. Dietas com inadequada quantidade de energia acarretam redução na produção de leite e causam excessiva perda de peso, prejudicando a reprodução e diminuindo a resistência dos animais a doenças. Dietas com excesso de energia aumentam os custos com alimentação e causam problemas metabólicos (Weiss, 1998).

A eficiência de produção pode ser expressa, entre outras formas, pela produção de leite corrigida (PLC) em relação ao consumo de energia líquida (CEL), ou seja, PLC/ CEL (Pantoja et al., 1995), e pela produção de leite corrigida em relação ao consumo de MS (PLC/CMS) (Grant \& Weidner, 1992). Jenkins (1999) utilizou dietas com 0, 1, 2, 3, 4 e 5\% de AG insaturados na dieta e observou que a eficiência, expressa como PLC/CMS, elevou linearmente com o aumento do nível de AG insaturados na dieta, enquanto o CMS diminuiu e a produção de leite permaneceu constante até os AG insaturados excederem 2\% da MS (depois deste nível, houve declínio na produção). Garcia-Bojalill et al. (1998) avaliaram a inclusão de dois níveis (0 e 2,2\%) de sais de cálcio de óleo de palma e observaram aumento de $7 \%$ na eficiência de produção (PLC/CMS) com a adição de óleo protegido à dieta.

No entanto, ainda são poucas as informações disponíveis sobre a eficiência do uso de fontes alternativas de lipídeos sobre a produção de leite. Usualmente, essas fontes são de baixo custo, o que aumenta o interesse em maximizar sua utilização em dietas para vacas leiteiras.

Objetivou-se com este trabalho avaliar os efeitos da suplementação de sais de cálcio de óleo de palma (GP), farelo de arroz integral + óleo de arroz (FAIO) ou farelo de arroz integral + sebo bovino (FAIS) na dieta sobre o balanço energético, o consumo de MS, a produção de leite e a eficiência produtiva de vacas leiteiras de alta produção no terço inicial da lactação.

\section{Material e Métodos}

O experimento foi conduzido no período de junho a setembro de 2000, no Sistema de Pecuária de Leite-SISPEL, na Estação Experimental Terras Baixas (ETB) da EMBRAPA Clima Temperado, localizada no município de Capão do Leão, RS.

Foram utilizadas oito vacas Jersey multíparas de $2^{2} \underline{a}$ e $3^{\text {a }}$ ordens de lactação, aos 70 dias de lactação, com 420 kg de PV e escore corporal de 3,5 a 4. O delineamento experimental utilizado constou de um quadrado latino 4 x 4 duplo, com quatro períodos experimentais de 21 dias (14 dias de adaptação às dietas e sete dias para coleta dos dados).

Os animais receberam dietas constituídas de volumoso (à base de silagem de milho e feno de alfafa), concentrado (composto de grão de milho moído, farelo de soja e farelo de trigo) e mistura mineral. Os tratamentos consistiram da inclusão de fontes de lipídeos no concentrado, conforme descrito a seguir: $\mathrm{C}$ = controle, sem adição de lipídeos; GP = inclusão de óleo protegido (sais de cálcio de óleo de palma); FAIO = adição de farelo de arroz integral (FAI) + óleo de arroz; FAIS = adição de FAI + sebo bovino. Ressalta-se que, na ocasião da execução deste trabalho, ainda não havia a proibição do uso do sebo na alimentação de ruminantes no Brasil (Instrução Normativa no 8 de 25 de março de 2004 do Ministério da Agricultura, Pecuária e do Abastecimento) e que sua utilização na dieta teve como 
propósito reduzir o grau de insaturação do óleo do farelo de arroz integral.

As vacas foram ordenhadas duas vezes ao dia (às $7 \mathrm{e}$ 17h) em uma sala de ordenha do tipo espinha de peixe, duplo quatro. O leite produzido foi pesado e as produções foram registradas diariamente para o cálculo da eficiência de produção, realizado nos últimos sete dias de cada período experimental. As amostras de leite foram coletadas nos dois últimos dias do período de coleta, após as ordenhas (matutinas e vespertinas) e utilizadas para formar uma amostra composta diária individual proporcional às ordenhas do dia. As análises do leite foram feitas no leite resfriado, no dia posterior ao da coleta, para determinação da porcentagem de gordura.

A produção de leite corrigida para 3,5\% de gordura (PLC3,5\%) foi calculada pela fórmula: PLC3,5\%=[(0,4324x kg de leite $)+(16,216 x$ kg de gordura) (Bremmer et al., 1997).

As vacas foram pesadas no primeiro, segundo, penúltimo e último dias de cada período de coleta, após a ordenha da manhã, antes da alimentação.

O concentrado foi fornecido três vezes ao dia e o volumoso duas vezes ao dia, após homogeneização, permitindo-se aproximadamente $10 \%$ de sobras. Diariamente, procedeu-se ao registro da quantidade de alimento consumido e à amostragem dos alimentos fornecidos e das sobras. Amostras diárias foram misturadas para formação de amostras compostas por tratamento e por período. As amostras compostas foram acondicionadas em sacos plásticos, identificadas por tratamento, por período e por animal e armazenadas em freezer a $-18^{\circ} \mathrm{C}$ para posteriores análises bromatológicas. Ao final de cada período experimental, as amostras da silagem de milho, do feno de alfafa e das sobras foram descongeladas, pré-secas em estufa de circulação forçada $\left(55^{\circ} \mathrm{C}\right.$ por 72 horas) e, posteriormente, trituradas em moinho tipo Willey com peneira de 30 mesh para análise e determinação dos teores de MS, PB, EE e cinzas utilizando-se as técnicas descritas por Silva \& Queiroz (2002). As concentrações de FDN foram determinadas segundo Van Soest et al. (1991). Os carboidratos não-estruturais foram calculados pela fórmula: $\mathrm{CNE}=100-(\% \mathrm{FDN}+\% \mathrm{~PB}+\% \mathrm{EE}+$ \%cinzas), segundo Sniffen et al. (1992). Foram feitas amostras compostas do concentrado por tratamento, contudo, para o volumoso oferecido e as sobras, obteve-se uma amostra composta por período de coleta. A composição químico-bromatológica dos ingredientes, a composição percentual e a composição químico-bromatológica das dietas experimentais encontram-se, respectivamente, nas Tabelas 1,2 e 3.

Para estimativa da energia líquida (EL) dos suplementos lipídicos, foram utilizadas as seguintes equações do NRC
Tabela 1 - Composição químico-bromatológica dos ingredientes incorporados às dietas experimentais (\%MS)

Table 1 - Chemical composition of ingredients incorporated into experimental diets (\% DM)

\begin{tabular}{|c|c|c|c|c|c|}
\hline \multirow[t]{3}{*}{$\begin{array}{l}\text { Ingrediente } \\
\text { Ingredient }\end{array}$} & \multicolumn{5}{|c|}{$\begin{array}{c}\text { Nutriente } \\
\text { Nutrient }\end{array}$} \\
\hline & MS (\%) & $\mathrm{PB}$ & $\mathrm{EE}$ & FDN & CNE \\
\hline & & CP & & $N D F$ & $N S C$ \\
\hline & \multicolumn{5}{|c|}{$\%$ MS (\% DM) } \\
\hline $\begin{array}{l}\text { Feno de alfafa } \\
\text { Alfalfa hay }\end{array}$ & 80,63 & 19,46 & 2,49 & 44,04 & 9,85 \\
\hline $\begin{array}{l}\text { Silagem de milho } \\
\text { Corn silage }\end{array}$ & 29,56 & 8,63 & 3,10 & 47,06 & 6,42 \\
\hline $\begin{array}{l}\text { Milho grão } \\
\text { Corn grain }\end{array}$ & 88,00 & 9,03 & 4,91 & 16,19 & 1,90 \\
\hline $\begin{array}{l}\text { Farelo de soja } \\
\text { Soybean meal }\end{array}$ & 90,00 & 48,00 & 1,04 & 11,93 & 7,30 \\
\hline $\begin{array}{l}\text { Farelo de trigo } \\
\text { Wheat bran }\end{array}$ & 90,00 & 17,10 & 4,40 & 51,00 & 6,90 \\
\hline $\begin{array}{l}\text { Óleo de arroz } \\
\text { Rice oil }\end{array}$ & 100,00 & - & 100,00 & - & - \\
\hline $\begin{array}{l}\text { Farelo de arroz } \\
\text { integral } \\
\text { Whole rice bran }\end{array}$ & 91,00 & 12,89 & 16,60 & 22,73 & 12,80 \\
\hline $\begin{array}{l}\text { Whole rice bran } \\
\text { Sebo bovino } \\
\text { Bovine tallow }\end{array}$ & 100,00 & - & 99,50 & - & - \\
\hline $\begin{array}{l}\text { Sais de cálcio de } \\
\text { óleo de palma }\end{array}$ & 86,00 & - & 82,00 & - & 10,00 \\
\hline
\end{tabular}

(2001): EL(total) $=[\mathrm{EL}($ mant. $)+\mathrm{EL}($ prod. leite $)+\mathrm{EL}($ ganho de peso)]; em que EL(mant.) = energia líquida para mantença $(\mathrm{Mcal} / \mathrm{kg}) ; \mathrm{EL}($ prod. leite) = energia líquida para produção de leite (Mcal/dia); e EL(ganho de peso) = energia líquida para ganho de peso (Mcal/dia). Todas essas variáveis foram calculadas como a seguir: $\mathrm{EL}$ (mant.) $=0,08 \times \mathrm{PV} 0,75$ utilizando-se a média de PV ( $\mathrm{kg})$ dos animais em cada dieta: EL(prod. leite) $=0,74 \times$ PLC4\%.

As exigências de EL(ganho de peso) foram estimadas como 5,12 Mcal de EL/kg de PV ganho ou-4,92 Mcal de EL/ kg de PV perdido utilizando-se a média da mudança de PV (kg) em cada tratamento.

O balanço energético foi calculado segundo Lucy et al. (1992), pela seguinte fórmula: BE = EL(cons.) - EL(mant.) EL(leite), em que: EL(cons.) = energia líquida consumida (Mcal/dia), estimada como o CMS(kg/dia) multiplicado pela EL da dieta (Mcal/dia); EL(mant.) = energia líquida para mantença (Mcal $/ \mathrm{kg})$, calculada como $\mathrm{PV}^{0,75}$ multiplicado por 0,08; EL(leite) = energia líquida do leite (Mcal/dia), calculada como produção de leite (libras) x [(41,84 x \% gordura do leite $)+(22,29 \mathrm{x} \%$ sólidos totais desengordurados $)$ -25,58/100]

As variáveis relacionadas à produção e ao consumo de nutrientes foram submetidas à análise de variância e as médias foram comparadas pelo teste Tukey, utilizando-se o 
Tabela 2 - Composição das dietas experimentais (\% MS) Table 2 - Composition of experimental diets (\% DM)

\begin{tabular}{|c|c|c|c|c|}
\hline \multirow[t]{2}{*}{$\begin{array}{l}\text { Ingrediente } \\
\text { Ingredient }\end{array}$} & \multicolumn{4}{|c|}{$\begin{array}{l}\text { Dieta }^{1} \\
\text { Diet }\end{array}$} \\
\hline & $\begin{array}{l}\mathrm{C} \\
\mathrm{C}\end{array}$ & $\begin{array}{l}\text { GP } \\
P F\end{array}$ & $\begin{array}{l}\text { FAIO } \\
\text { WRBO }\end{array}$ & $\begin{array}{l}\text { FAIS } \\
\text { WRBT }\end{array}$ \\
\hline $\begin{array}{l}\text { Feno de alfafa } \\
\text { Alfalfa hay }\end{array}$ & 26,65 & 26,26 & 25,65 & 26,40 \\
\hline $\begin{array}{l}\text { Silagem de milho } \\
\text { Corn silage }\end{array}$ & 36,02 & 35,49 & 34,67 & 35,68 \\
\hline $\begin{array}{l}\text { Milho grão } \\
\text { Corn grain }\end{array}$ & 22,64 & 18,10 & 11,53 & 14,39 \\
\hline $\begin{array}{l}\text { Farelo de soja } \\
\text { Soybean meal }\end{array}$ & 13,10 & 13,43 & 12,30 & 12,44 \\
\hline $\begin{array}{l}\text { Farelo de trigo } \\
\text { Wheat bran }\end{array}$ & - & 2,15 & - & - \\
\hline $\begin{array}{l}\text { Óleo de arroz } \\
\text { Rice oil }\end{array}$ & - & - & 0,58 & - \\
\hline $\begin{array}{l}\text { Farelo de arroz integral } \\
\text { Whole rice bran }\end{array}$ & - & - & 13,49 & 7,57 \\
\hline $\begin{array}{l}\text { Sebo bovino } \\
\text { Bovine tallow }\end{array}$ & - & - & - & 1,41 \\
\hline $\begin{array}{l}\text { Sais de cálcio de óleo de palma } \\
\text { Calcium salts of palm oil }\end{array}$ & - & 3,01 & - & - \\
\hline $\begin{array}{l}\text { Calcário calcítico } \\
\text { Limestone }\end{array}$ & 0,37 & 0,36 & 1,05 & 0,91 \\
\hline $\begin{array}{l}\text { Suplemento mineral }{ }^{2} \\
\text { Mineral supplement }\end{array}$ & 0,36 & 0,36 & 0,35 & 0,36 \\
\hline $\begin{array}{l}\text { Fosfato bicálcico } \\
\text { Dicalcium phosphate }\end{array}$ & 0,86 & 0,84 & 0,83 & 0,84 \\
\hline
\end{tabular}

${ }^{1} \mathrm{C}$ - controle, sem suplementação com lipídeos; GP - óleo protegido (sais de cálcio de óleo de palma); FAIO - farelo de arroz integral (FAI) + óleo de arroz; FAIS - FAI + sebo bovino.

${ }^{1} \mathrm{C}$ - control, without fat supplementation; PF - protected oil (calcium salts of palm oil); WRBO - whole rice bran (WRB) + rice oil and); WRBT - whole rice bran + bovine tallow.

2 Composição por $\mathrm{kg}$ de produto (composition per $\mathrm{kg}$ of product): $\mathrm{Ca}-230 \mathrm{~g}$; P - 90 g; S - 15 g; Mg - 20 g; Cu - 700 mg; Zn - 2.700 mg; Co - 100 mg; I - 80 mg; Se - 20 mg; Fe - 2.000 mg; excipiente (excipient) q.s.p. - 1.000 g.

Tabela 3 - Composição químico-bromatológica das dietas experimentais (\% MS)

Table 3 - Chemical composition of experimental diets (\% DM)

\begin{tabular}{|c|c|c|c|c|}
\hline \multirow[t]{3}{*}{$\begin{array}{l}\text { Ingrediente } \\
\text { Ingredient }\end{array}$} & \multicolumn{4}{|c|}{$\begin{array}{l}\text { Dieta }^{1} \\
\text { Diet }\end{array}$} \\
\hline & C & GP & FAIO & FAIS \\
\hline & C & $P F$ & WRBO & WRBT \\
\hline & & & & \\
\hline \multirow[t]{2}{*}{ MS (DM) } & 50,33 & 52,37 & 50,29 & 52,16 \\
\hline & \multicolumn{4}{|c|}{$(\% \mathrm{MS})(\% D M)$} \\
\hline MO (OM) & 92,24 & 92,48 & 90,84 & 91,40 \\
\hline $\mathrm{PB}(C P)$ & 16,64 & 16,10 & 17,04 & 16,01 \\
\hline FDN (NDF) & 33,20 & 33,92 & 34,77 & 33,04 \\
\hline $\mathrm{EE}$ & 2,99 & 5,13 & 5,29 & 5,16 \\
\hline CNE (NSC) & 38,64 & 35,22 & 35,83 & 34,41 \\
\hline $\mathrm{Ca}$ & 0,94 & 0,90 & 1,07 & 1,08 \\
\hline \multirow[t]{2}{*}{$\mathrm{P}$} & 0,48 & 0,47 & 0,59 & 0,56 \\
\hline & \multicolumn{4}{|c|}{ (Mcal/d) } \\
\hline $\begin{array}{l}\text { Energia líquida }(\mathrm{Mcal} / \text { dia })^{1} \\
\text { Net energy }(\text { Mcal/day) }\end{array}$ & 28,07 & 31,10 & 29,62 & 29,18 \\
\hline
\end{tabular}

Statistical Analysis System (SAS , 2000), conforme o modelo matemático a seguir:

Yijkl = M + QLi + Pj (QL) + Vk (QL) + Tz + QL + Tiz + Eijki em que: Yijkl = valor da variável obtida em cada observação; $\mathrm{M}=$ média geral da variável no experimento; QLi = efeito do quadrado latino $(\mathrm{GL}=1)$; $\mathrm{Pj}(\mathrm{QL})=$ efeito do período dentro do quadrado latino $(\mathrm{GL}=6) ; \mathrm{Vk}(\mathrm{QL})=$ efeito do animal dentro do quadrado latino $(\mathrm{GL}=6) ; \mathrm{Tz}=$ efeito de tratamento $(G L=3) ; Q L+T i z=$ efeito da interação quadrado latino $\times$ tratamento $(\mathrm{GL}=3)$; Eijki = erro experimental $(\mathrm{GL}=12)$.

\section{Resultados e Discussão}

Como demonstrado na Tabela 4, a suplementação com lipídeos ocasionou aumento médio de 2,0 kg/dia na PLC3,5\% ( $\mathrm{P}<0,05)$. Esse aumento, no entanto, não diferiu entre as fontes testadas. Do mesmo modo, Wu et al. (1993), utilizando 2,5\% de gordura proveniente de sais de cálcio de óleo de palma, sebo bovino ou prilled fat, verificaram aumento de 2,01 kg de leite dia em comparação ao tratamento controle.

Segundo Kronfeld (1982), a quantidade de glicose na glândula mamária é determinante do volume de leite produzido. O aumento da extração de AGCL do sangue pela glândula mamária decresce a síntese de AG de cadeias curta e média. Essa síntese requer a utilização de glicose como fornecedora de agentes redutores NADPH, via ciclo das pentoses. A utilização de lipídeos dietéticos pode propiciar a redução na utilização da glicose (Cant et al., 1993), disponibilizando maior quantidade de glicose para outros processos metabólicos como a produção de leite (Palmquist \& Jenkins, 1980).

O consumo de MS, expresso em kg/dia e em \%PV, não diferiu entre as dietas. Canale et al. (1990) também não encontraram diferença significativa para o consumo de MS (kg/dia, ou \%PV) ao utilizarem 2,56\% de sais de cálcio de óleo de palma na dieta de vacas lactantes.

Considerando esses resultados, pode-se inferir que a maior produção de leite nos animais sob suplementação com lipídeos foi ocasionada pela direta incorporação dos AG pré-formados da dieta na gordura do leite, o que aumentou a eficiência da secreção de gordura. A ausência de efeitos da suplementação na dieta sobre o consumo de MS pode ter resultado da não-influência dos lipídeos da dieta sobre os microrganismos ruminais. De acordo com Wu et al. (1993), a diminuição do consumo de MS com a adição de fontes de lipídeos pode ocorrer no início da lactação, quando o tecido adiposo libera AG, que, juntamente com os AG de origem dietética, podem exceder a quantidade de gordura que o animal pode utilizar eficientemente, ocasionando diminuição 
Tabela 4 - Valores médios de produção de leite corrigida para 3,5\% de gordura (PLC3,5\%) e consumo MS obtidos com as dietas experimentais

Table 4 - Means values of 3.5\% fat correct milk (3.5\% FCM) and DM intake (DMI) obtained with the experimental diets

\begin{tabular}{|c|c|c|c|c|c|c|}
\hline \multirow[t]{2}{*}{ Item $^{2}$} & \multicolumn{4}{|c|}{$\begin{array}{c}\text { Dieta }^{1} \\
\text { Diet }^{1}\end{array}$} & \multirow[t]{2}{*}{$\mathrm{P}>\mathrm{F}$} & \multirow[t]{2}{*}{$\mathrm{CV} \%$} \\
\hline & $\mathrm{C}$ & GP & FAIO & FAIS & & \\
\hline PLC3,5\% (L/d) $(3.5 \%$ FCM $)$ & $19,81 b$ & $22,24 \mathrm{a}$ & $21,73 a$ & $21,65^{\mathrm{a}}$ & 0,002 & 4,34 \\
\hline Consumo de MS (kg/d) (DM intake) & $15,87 a$ & $16,39 a$ & $16,05 \mathrm{a}$ & $15,99^{\mathrm{a}}$ & 0,431 & 3,97 \\
\hline Consumo de MS/PV (\%) (DM intake/BW) & 3,81a & 3,95a & $3,84 a$ & $3,79^{a}$ & 0,222 & 4,02 \\
\hline
\end{tabular}

${ }^{1}$ C - Controle, sem suplementação com lipídeos; GP - óleo protegido (sais de cálcio de óleo de palma); FAIO - farelo de arroz integral (FAI) + óleo de arroz; FAIS - FAI + sebo bovino

2 Médias, na linha, seguidas de diferentes letras na linha diferem $(P<0,05)$ pelo teste Tukey.

${ }^{1} \mathrm{C}$ - control, without fat supplementation; PF - protected oil (calcium salts of palm oil); WRBO - whole rice bran (WRB) + rice oil and); WRBT - whole rice bran + bovine tallow.

2 Means, within a row, followed of different letters in a row differ $(P<0.05)$ by Tukey.

Tabela 5 - Energia líquida do leite obtida em relação ao consumo de energia líquida (EL (leite) $/$ Consumo de EL) e produção de leite corrigida para 3,5\% de gordura em relação ao consumo de MS (PLC3,5\%/CMS), ao consumo de energia líquida (PLC3,5\%/CEL) e ao peso metabólico (PLC3,5\%/PM),

Table 5 - Net energy of milk obtained in relation to net energy intake ( $N E_{(\text {milk })} / \mathrm{NE}$ intake), milk production of $3.5 \%$ fat correct milk in function of DM intake (3.5\% FCM/DMI), net energy intake (3.5\% FCM/ NEI) and metabolic weight (3.5\% FCM/MW)

\begin{tabular}{|c|c|c|c|c|c|c|}
\hline \multirow[t]{3}{*}{ Item } & \multicolumn{4}{|c|}{$\begin{array}{c}\text { Dieta }^{1} \\
\text { Diet }^{1}\end{array}$} & \multirow[t]{3}{*}{$\mathrm{P}>\mathrm{F}$} & \multirow[t]{3}{*}{ CV\% } \\
\hline & $\mathrm{C}$ & GP & FAIO & FAIS & & \\
\hline & C & $P F$ & $W R B O$ & WRBT & & \\
\hline $\mathrm{IL}_{(\text {leite) }} / \mathrm{CEL}(\%)\left(N E_{(m i l k)} / N E I\right)$ & $56,53 a$ & $54,54 a$ & $57,18 \mathrm{a}$ & $55,80 a$ & 0,497 & 6,24 \\
\hline PLC3,5\%/Consumo de MS (kg/kg) (3.5\% FCM/DM intake) & $1,25 a$ & $1,36 a$ & $1,35 a$ & $1,35 \mathrm{a}$ & 0,130 & 7,76 \\
\hline PLC3,5\%/Consumo de EL ( $\mathrm{kg} / \mathrm{Mcal})$ (3.5\% FCM/NE intake) & $0,813 \mathrm{a}$ & $0,799 a$ & $0,824 \mathrm{a}$ & $0,816 \mathrm{a}$ & 0,783 & 6,04 \\
\hline
\end{tabular}

${ }^{1}$ C - Controle, sem suplementação com lipídeos; GP - óleo protegido (sais de cálcio de óleo de palma); FAIO - farelo de arroz integral (FAI) + óleo de arroz; FAIS - FAI + sebo bovino

Médias, na linha, seguidas de diferentes letras na linha diferem $(P<0,05)$ pelo teste Tukey.

${ }^{1}$ C - control, without fat supplementation; PF - protected oil (calcium salts of palm oil); WRBO - whole rice bran (WRB) +rice oil and); WRBT - whole rice bran + bovine tallow Means, within a row, followed of different letters in a row differ $(P<0.05)$ by Tukey.

do consumo. Como no começo do experimento as vacas se encontravam, aproximadamente, no 70 을 dia pós-parto, a suplementação com lipídeos não foi feita concomitantemente à mobilização das reservas corporais para produção de leite.

A eficiência, expressa em energia líquida do leite (EL(leite)) em relação à energia líquida consumida (CEL), não foi influenciada pela suplementação e não diferiu entre as fontes de lipídeos utilizadas (Tabela 5). Smith et al. (1978), utilizando uma dieta controle (2,9\% EE), uma com teor médio de gordura (8,4\% EE) e outra com alto teor de gordura (14,3\% EE), verificaram valores de eficiência energética de 55,6; 65,8 e 66,1\%, respectivamente, próximos ao encontrado nesse trabalho.

Não houve efeito da suplementação de lipídeos sobre a conversão da MS consumida para PLC3,5\% (Tabela 5). Por outro lado, Canale et al. (1990) verificaram aumento na conversão de MS consumida para PLC4\% quando as vacas lactantes foram alimentadas com 2,56\% de sais de óleo de palma em comparação a uma dieta controle. Os resultados encontrados nesse experimento para a eficiência de utilização dos alimentos para produção de leite, em kg de leite por kg de MS consumida, foram próximos aos relatados por Wu et al. (1993), que, ao testarem uma dieta controle com $3,7 \%$ de EE e três dietas suplementadas com sebo bovino (6,2\% EE), sais de cálcio de óleo de palma (6,1\% EE) ou prilled fat (6,2\% de EE), encontraram eficiência (PLC3,5\%/CMS) de 1,25 a 1,36.

A eficiência expressa como PLC3,5\% não diferiu entre os consumos de EL consumida, mas houve diferença quanto à eficiência de PLC3,5\% em relação ao PM, observando-se superioridade dos animais sob suplementação com lipídeos em comparação aos da dieta controle, independentemente da fonte de óleo utilizada (Tabela 5). Canale et al. (1990) verificaram valores para PLC4\%/PM de 0,213 e 0,221 nas dietas controle e com inclusão de sais de cálcio de óleo de palma, respectivamente. Palmquist \& Conrad (1978) encontraram valores de 0,202; 0,221; 0,216 e 0,240 kg PLC4\%/PM, respectivamente, para as dietas controle $(3,18 \% \mathrm{EE})$, com 
Tabela 6 - Balanço energético (BE), consumo de $E L$, energia líquida de mantença $\left(\mathrm{EL}_{(\text {mant.) }}\right)$ e energia líquida do leite produzido ( $\left.E L_{(\text {leite) }}\right)$ Table 6 - Energy balance $(E B)$, net energy intake (NEI), net energy for maintenance $\left(N E_{(\text {maint.) }}\right)$ and net energy for milk yield ( $\left.N E_{(m i l k)}\right)$

\begin{tabular}{|c|c|c|c|c|c|c|}
\hline \multirow[t]{2}{*}{ Item $^{2}$} & \multicolumn{4}{|c|}{$\begin{array}{c}\text { Dieta }^{1} \\
\text { Diet }^{1}\end{array}$} & \multirow[t]{2}{*}{$\mathrm{P}>\mathrm{F}$} & \multirow[t]{2}{*}{$\mathrm{CV} \%$} \\
\hline & $\mathrm{C}$ & GP & FAIO & FAIS & & \\
\hline $\mathrm{BE}(E B)$ & 3,163b & 5,329a & 3,952ab & 4,234ab & 0,016 & 26,80 \\
\hline CEL (Mcal/d) (NEI) & $24,461 c$ & $27,920 a$ & $26,448 b$ & $26,546 b$ & 0,001 & 3,19 \\
\hline $\mathrm{EL}_{\text {(mant.) }}(\mathrm{Mcal} / \mathrm{d})\left(N E_{\text {[maint }]}\right)$ & 7,406a & 7,385a & 7,413a & 7,459a & 0,547 & 1,37 \\
\hline
\end{tabular}

${ }^{1}$ C - Controle, sem suplementação com lipídeos; GP - óleo protegido (sais de cálcio de óleo de palma); FAIO - farelo de arroz integral (FAI) + óleo de arroz; FAIS - FAI + sebo bovino

2 Médias, na linha, seguidas de diferentes letras na linha diferem $(P<0,05)$ pelo teste Tukey.

${ }^{1} \mathrm{C}$ - control, without fat supplementation; PF - protected oil (calcium salts of palm oil); WRBO - whole rice bran (WRB) +rice oil and); WRBT - whole rice bran + bovine tallow.

${ }^{2}$ Means, within a row, followed of different letters in a row differ $(P<0.05)$ by Tukey.

inclusão de soja crua (5,93\%EE), de óleo de soja (5,7\% EE) e gordura (10,8\% EE). Esses valores, no entanto, foram inferiores aos encontrados por Duarte et al. (2005), que, utilizando uma dieta controle com 3,63\% de EE e três com suplementação de lipídeos na forma de sebo bovino $(6,28$ EE), sais de cálcio de óleo de palma (6,29\%) e grão de soja integral moído (6,28\%), verificaram PLC4\%/PM de 0,273; 0,$275 ; 0,300 ; 0,270$, respectivamente.

Os valores médios encontrados para o balanço energético (BE), o consumo de EL, a energia líquida de mantença (EL(mant.)) e a energia líquida do leite produzido (EL(leite)) podem ser observados na Tabela 6.

O consumo de EL na dieta com óleo protegido foi maior quando os animais foram alimentados com as dietas com farelo de arroz integral + óleo de arroz e com farelo de arroz integral + sebo (FAIO e FAIS), provavelmente porque os sais de cálcio de óleo de palma utilizados na dieta possuem EL de 6,52 Mcal/kg (Andrew et al., 1991), valor superior à EL dos suplementos lipídicos utilizados nas dietas FAIO e FAIS. Não foram observadas diferenças significativas entre as dietas C, FAIO e FAIS.

O balanço energético obtido com a dieta com sais de cálcio de óleo de palma (GP) foi superior ao observado com a dieta controle, porém semelhante ao encontrado quando fornecidas as dietas FAIO e FAIS. Não foram observadas diferenças significativas no balanço energético obtido com as dietas controle, FAIO e FAIS. A superioridade do balanço energético obtido com a dieta GP em relação ao da dieta controle pode estar relacionada ao maior consumo de EL quando os animais foram alimentados com essa dieta.

Os valores de energia líquida nas fontes de lipídeos foram 5,07; 4,37 e 4,33 Mcal/kg, respectivamente, para os sais de cálcio de óleo de palma, FAI + óleo de arroz (79,52\% EE do FAI + 20,48\% EE do óleo de arroz) e FAI + sebo bovino (45,51\% EE do FAI + 54,49\% EE do sebo) (Tabela 7). A estimativa da EL para os sais cálcio de óleo de palma foi menor que os $6,52 \mathrm{Mcal} / \mathrm{kg}$ estimados por Andrew et al. (1991) e que os 5,69 Mcal/kg estimados por Holter et al. (1992), mas superior aos 4,61 Mcal/kg estimados por $\mathrm{Wu}$ et al. (1993). Andrew et al. (1991) avaliaram a EL dos sais de cálcio de óleo de palma em dois níveis de proteína na dieta (16 e 20\% de PB) e encontraram valores de 7,53 e 5,52 $\mathrm{Mcal} / \mathrm{kg}$, respectivamente, nesta fonte de óleo. Segundo Wu et al. (1993), a considerável variação da EL dos sais de cálcio de óleo de palma ocorre porque esse suplemento

Tabela 7 - Estimativa da energia líquida de lactação total $\left(\mathrm{EL}_{(\text {total })}\right)$, porcentagem de ácidos graxos na dieta (AG\%), porcentagem de lipídeo nos suplementos da dieta, consumo de MS, consumo de lipídeo dos suplementos e energia líquida dos suplementos (EL suplemento) das dietas experimentais

Table 7 - Estimation of total net energy of lactation ( $\left.N E_{L(\text { total) }}\right)$, percentage of dietary fatty acid (FA\%), percentage of dietary fat from supplement, dry matter intake (DMI), fat intake from supplements and netenergy of supplements (NE supplement) of experimental diets

\begin{tabular}{|c|c|c|c|c|}
\hline \multirow[t]{3}{*}{$\begin{array}{l}\text { Ingrediente } \\
\text { Ingredient }\end{array}$} & \multicolumn{4}{|c|}{$\begin{array}{c}\text { Dieta }^{1} \\
\text { Diet }\end{array}$} \\
\hline & C & GP & FAIO & FAIS \\
\hline & C & $P F$ & WRBO & WRBT \\
\hline $\begin{array}{l}\mathrm{EL}_{(\text {total }}{ }^{2}(\mathrm{Mcal} / \mathrm{d}) \\
N E_{\text {Lftotal) }}\end{array}$ & 21,06 & 22,61 & 22,35 & 22,29 \\
\hline $\begin{array}{l}\text { AG na dieta(\%) } \\
\text { FA in the diet }\end{array}$ & 3,09 & 5,60 & 5,68 & 5,67 \\
\hline $\begin{array}{l}\text { Lipídeo do suplemento (\%) } \\
\text { Fat from supplement }\end{array}$ & - & 2,51 & 2,59 & 2,58 \\
\hline $\begin{array}{l}\text { Consumo de MS (kg/d) } \\
\text { DM intake }\end{array}$ & - & 16,39 & 16,05 & 15,99 \\
\hline $\begin{array}{l}\text { Consumo de lipídeo do } \\
\text { suplemento }(\mathrm{kg} / \mathrm{d})\end{array}$ & - & 0,41 & 0,42 & 0,41 \\
\hline $\begin{array}{l}\text { Fat intake from supplement } \\
\left.\text { EL do suplemento }{ }^{3} \text { (Mcal } / \mathrm{kg}\right) \\
N E \text { of supplement }\end{array}$ & - & 5,07 & 4,37 & 4,33 \\
\hline
\end{tabular}

${ }^{1} \mathrm{C}$ - Controle, sem suplementação com lipídeos; GP - óleo protegido (sais de cálcio de óleo de palma); FAIO - farelo de arroz integral (FAI) + óleo de arroz; FAIS - FAI + sebo bovino.

${ }^{1} \mathrm{C}$ - control, without fat supplementation; PF - protected oil (calcium salts of palm oil); WRBO - whole rice bran (WRB) + rice oil and); WRBT - whole rice bran + bovine tallow.

2 Estimada de acordo com o NRC (2001) (Estimated according to NRC, 2001).

${ }^{3}$ Estimada de acordo com Wu et al. (1993) (Estimated according to Wu et al., 1993). 
participa em proporção muito reduzida (2,95\%) na dieta total e porque esses valores derivam da estimativa da EL de todos os alimentos que compõem a dieta. O resultado obtido para a EL com a dieta FAI foi 1,8 Mcal/kg (média da estimativa dos valores de EL das dietas FAI, FAIO e FAIS, Tabela 8), próximo ao de 1,6 Mcal/kg estimado pelo NRC (2001).

De acordo com Weiss (1998), as variações nos valores de EL dos alimentos podem ser explicadas por variações normais na utilização de energia pelas vacas, por diferenças entre experimentos causadas pelo desenho experimental, por fatores relacionados ao animal (consumo de MS e homogeneidade dos animais), por fatores relacionados à dieta (fonte e quantidade de PB) e por erros analíticos (medida da variação do PV). Dados reportados por Nörnberg et al. (2004) comprovaram que a fonte de energia afeta a eficiência energética. O amido e a fibra têm efeito negativo, enquanto o lipídeo tem efeito positivo na eficiência energética, pois não promove perda de energia pela produção de metano ou energia urinária (Andrew et al., 1991).

Tabela 8 - Energia líquida de lactação do lipídeo dos suplementos (EL $\left.L_{\text {Lactação }}\right)$ farelo de arroz integral (FAl) e sebo bovino e energia líquida do FAI nas dietas farelo de arroz integral + óleo de arroz (FAIO) e com farelo de arroz integral + sebo bovino (FAIS)

Table 8 - $\quad$ Net energy of lactation from fat in the supplement ( $\left.N E_{\text {Lactation }}\right)$, whole rice bran (WRB), bovine tallow, net energy of WRB in the diets with whole rice bran and rice oil (WRBO) and whole rice bran and bovine tallow (WRBT)

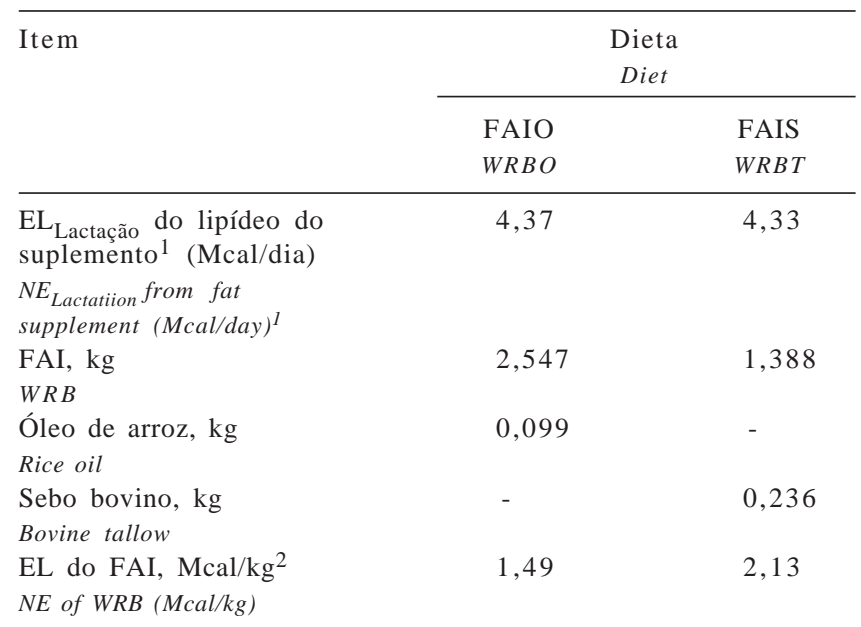

${ }^{1}$ Estimada de acordo com Wu et al. (1993).

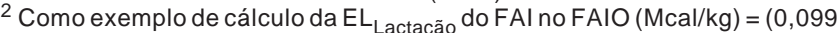
$x E L$ do óleo de arroz $)+(2,547 \times E L$ do $F A I)=4,37$. Para a $E L$ do óleo de arroz (FAIO) e EL do sebo bovino (FAIS), utilizou-se o valor de 5,84 Mcal/ $\mathrm{kg}$ (NRC, 2001).

${ }^{1}$ Estimated from according to Wu et al. (1993).

2 As example of calculation of $N E_{L}$ of WRB in WRBO $(\mathrm{Mcal} / \mathrm{kg})=\left(0.099 \times N E_{L}\right.$ of oil rice $)$ $+\left(2.547 \times N E_{L}\right.$ of $\left.W R B\right)=4.37$. $N E_{L}$ of oil rice $(W R B O)$ and $N E_{L}$ of bovine tallow $(W R C T)$ was used $5.84 \mathrm{Mcal} / \mathrm{kg}$ (NRC, 2001).

\section{Conclusões}

A utilização de sais de cálcio de óleo de palma, farelo de arroz integral + óleo de arroz ou farelo de arroz integral + sebo bovino na dieta (5\% de extrato etéreo na matéria seca) de vacas leiteiras no terço inicial da lactação aumentou a produção de leite corrigida para 3,5\% de gordura e não influenciou o consumo dos alimentos. A eficiência produtiva, expressa como produção de leite corrigida para 3,5\% de gordura em relação ao peso metabólico e ao balanço energético, aumentou com a adição de fontes de lipídeos na alimentação de vacas leiteiras; no entanto, não houve diferenças significativas entre as fontes utilizadas. A eficiência energética, expressa em energia líquida do leite em relação ao consumo de energia líquida, e a eficiência de produção, expressa como produção de leite corrigida para 3,5\% de gordura em relação ao consumo de matéria seca, não foram influenciadas pela adição de lipídeos na dieta. O farelo de arroz integral pode ser utilizado com óleo de arroz ou com sebo na dieta de vacas lactantes no terço inicial de lactação com a finalidade de aumentar o consumo de energia líquida e o balanço energético.

\section{Literatura Citada}

ANDREW, S.M.; TYRRELL, H.F.; REYNOLDS, C.K. et al. Net energy for lactation of calcium salts of long-chain fatty acids for cows fed silage-based diets. Journal of Dairy Science, v.74, n.8, p.2588-2600, 1991.

BREMMER, D.R.; OVERTON, T.R.; CLARK, J.H. Production and composition of milk form Jersey cows administered bovine somatotropina and fed ruminally protected amino acids. Journal of Dairy Science, v.80, n.7, p.1374-1380, 1997.

CANALE, C.J.; BURGESS, P.L.; MULLER, L.D. et al. Calcium salts of fatty acids in diets that differ in neutral detergent fiber: Effect on lactation performance and nutrient digestibility. Journal of Dairy Science, v.73, n.4, p.1031-1038, 1990.

CANT, J.P.; DEPETERS, E.J.; BALDWIN, R.L. Mammary uptake of energy metabolites in dairy cows fed fat and its relationships to milk protein depression. Journal of Dairy Science, v.76, n.8, p.2254-2265, 1993.

DIJK, H.V.; O’DELL, G.D.; PERRY, P.R. Extruded versus ground soybeans of dairy cows in early lactation. Journal of Dairy Science, v.75, n.8, p.2043-2070, 1983.

DUARTE, L.M.; STUMPF, W.S.; FISCHER, V. et al. Efeito de diferentes fontes de gordura na dieta de vacas Jersey sobre o consumo, a produção e a composição do leite. Revista Brasileira de Zootecnia, v.34, n.6, p.2020-2028, 2005.

GARCIA-BOJALIL, C.M.; STAPLES C.R.; RISCO, C.A. et al. Protein degradability and calcium salts of long-chain fatty acids in the diets of lactating dairy cows: productive responses. Journal of Dairy Science, v.81, n.5, p.1374-1384, 1998.

GRANT, R.J.; WEIDNER, S.J. Effect of fat form whole soybeans on performance of dairy cows fed rations differing in the fiber level and particle size. Journal of Dairy Science, n.75, v.10, p.2742-2752, 1992.

HEMKEN, R.W. Loss of fat from dairy cows. Journal of Dairy Science, v.54, n.4, p.574-564, 1970.

HOLTER, J.B.; HAYES, H.H.; URBAN, W.E. et al. Energy balance and lactation respond in Holstein cows supplemented with 
cottonseed with or without calcium soap. Journal of Dairy Science, v.74, n.5, p.1480-1494, 1992.

JENKINS, T.C. Lactation performance and fatty acid composition of milk from Holstein cows fed 0 to $5 \%$ oleamide. Journal of Dairy Science, v.82, n.7, p.1525-1531, 1999.

JERRED, M.J.; CARROLL, D.J.; COMBS, D.K. et al. Effects of fat supplementation and immature alfalfa to concentrate ratio on lactation performance of dairy cattle. Journal of Dairy Science, n.73, v.10, p.2842-2854, 1990.

KRONFELD, D.S. Major metabolic determinants of volume, mammary efficiency and spontaneous ketosis in dairy cows. Journal of Dairy Science, v.65, n.11, p.2204-2212, 1982.

KRONFELD, D.S.; DONOGHUE, S.S.; NAYLOR, J.M. et al. Metabolic effects of feeding protected tallow to dairy cows. Journal of Dairy Science, v.63, n.4, p.545-552, 1980.

LUCY M.C.; STAPLES, C.R.; THACHER W.W. et al. Influence of diet composition, dry-matter intake, milk-production and energy-balance on time of postpartum ovulation and fertility in dairy-cows. Journal of Animal Production, v.54, n.6, p.323-331, 1992.

NATIONAL RESEARCH COUNCIL - NRC. Nutrient requirements of dairy cattle. 7.rev.ed.Washington, D.C.: National Academy Press, 2001. 408p.

NORNBERG, J.L.; STUMPF W.J.; LÓPEZ, J. et al. Valor do farelo de arroz integral como fonte de gordura na dieta de vacas Jersey na fase inicial de lactação: digestibilidade aparente de nutrientes. Revista Brasileira de Zootecnia, v.33, n.6, p.2412-2421, 2004. (supl. 3).

PALMQUIST, D.L. Use of fats in diets for lactating dairy cows. In: WISEMAN, J. (Ed.) Fats in animal nutrition. Boston: Butterworth, 1984. p.357-360.

PALMQUIST, D.L. Using fat to meet the energy needs of lactating cows. Columbus: Ohio State University, 1988. p.1-20.

PALMQUIST, D.L.; CONRAD, H.R. High fat rations for dairy cows. Effects on feed intake, milk and fat production, and plasma metabolites. Journal of Dairy Science, v.61, n.7, p.890-901, 1978.
PALMQUIST, D.L.; JENKINS, T.C. Fat in lactation rations: review. Journal of Dairy Science, v.63, n.1, p.1-14, 1980.

PANTOJA, J.; FIRKINS, J.L.; EASTRIDGE, M.L. Site of digestion and milk production by cows fed fats differing in saturation, etherification, and chain length. Journal of Dairy Science, v.78, n.10, p.2247-2258, 1995.

SCHAUFF, D.J.; ELLIOT, J.P.; CLARK, J.H. et al. Effects of feeding lactating dairy cows diets containing whole soybeans and tallow. Journal of Dairy Science, v.75, n.7, p.1923-1935, 1992.

SILVA, D.J.; QUEIROZ, A.C. Análises de alimentos: métodos químicos e biológicos. 3.ed. Viçosa, MG: Universidade Federal de Viçosa, 2002. 235p.

SMITH, N.E.; DUNKLEY, W.L.; FRANKE, A.A. Effects of feeding protected tallow to dairy cows in early lactation. Journal of Dairy Science, v.61, n.6, p.747-756, 1978.

SNIFFEN, C.J.; O'CONNOR, J.D.; Van SOEST, P.J. A net carbohydrate and protein system for evaluating cattle diets: II. Carbohydrate and protein availability. Journal of Animal Science, v.70, p.3562-3577, 1992.

STATISTICAL ANALYSIS SYSTEM - SAS. User's guide. Version 8.12. Cary: 2000. 1686p.

Van SOEST, P.J.; ROBERTSON, J.B.; LEWIS, B.A. Methods of dietary fiber, neutral detergent fiber, and nonstarch polysaccharides in relation to animal nutrition. Journal of Animal Science, v.74, n.10, p.3583-3597, 1991.

WEISS, W.P. Symposium: Energy availability - Estimating the available energy content of feeds for dairy cattle. Journal of Dairy Science, v.81, n.3, p.830-839, 1998.

WU, Z.; HUBER, J.T.; SLEIMAN, F.T. et al. Effect of three supplemental fat sources on lactation and digestion in dairy cows. Journal of Dairy Science, v.76, n.11, p.3562-3570, 1993. 